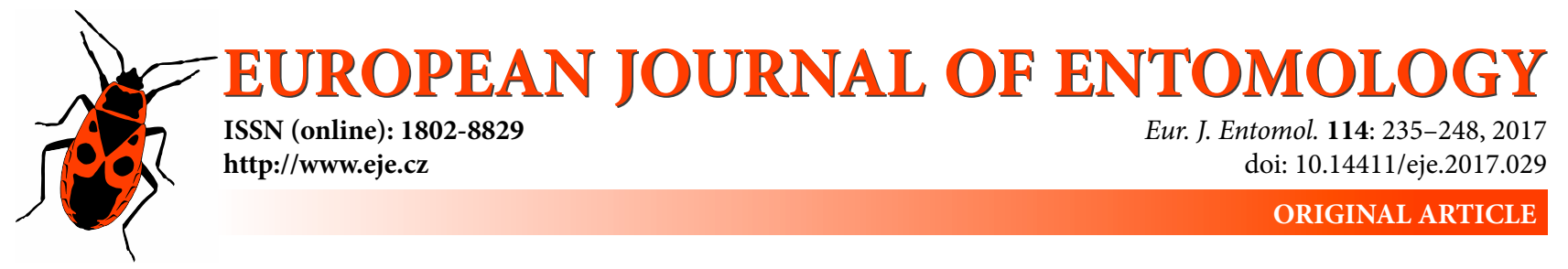

\title{
Juvenile hormone analogue, fenoxycarb, modulates ecdysone-triggered transcriptional hierarchy during programmed cell death of midgut in silkworm, Bombyx mori (Lepidoptera: Bombycidae)
}

\author{
Ebru GONCU, Ramazan URANLI and Osman PARLAK \\ Ege University, Faculty of Science, Department of Biology, 35100 Bornova - Izmir, Turkey; e-mails: ebru.goncu@ege.edu.tr, \\ uranli.ramazan@gmail.com, osman.parlak@ege.edu.tr
}

Key words. Lepidoptera, Bombycidae, apoptosis, autophagy, Bombyx mori, ecdysone related genes, juvenile hormone, midgut

\begin{abstract}
Steroid hormone 20-hydroxyecdysone and the sesquiterpenoid juvenile hormone are the main regulators of insect development; however, it is unclear how they interact in the regulation of metamorphic events. Using the silkworm, Bombyx mori, we show that the juvenile hormone analogue fenoxycarb affects the cascade of ecdysone regulated genes that control the programmed cell death in the larval midgut. Morphological changes that occur during cell death were investigated by studying crosssections of the midgut stained with hematoxylin and eosin. Apoptosis-specific DNA fragmentation was detected using TUNEL assay. Expression patterns of genes ATG8 and ATG12, which were used as indicators of autophagy, and genes of the ecdysoneregulated gene cascade were examined using real-time quantitative polymerase chain reaction. Fenoxycarb application on day 0 of the 5 th larval instar extended the feeding period and postponed programmed cell death in mature larval midgut. This effect was probably due to a delay in ecdysone secretion and associated changes in gene expression were mostly not a direct response to the fenoxycarb. However, differences in the gene expression patterns in the control and fenoxycarb treated insects during the prepupal and early pupal stages indicated that fenoxycarb may also exert a more direct effect on some genes of the ecdysone regulated gene cascade.
\end{abstract}

\section{INTRODUCTION}

Midgut remodeling in holometabolous insects, involves both programmed cell death (PCD) of mature larval midgut cells and formation of the pupal midgut from stem cells (Lee et al., 2002). Both of these events are strictly controlled by the insect steroid hormone 20 -hydroxyecdysone (20E), sesquiterpenoid juvenile hormone (JH) and some paracrine factors (Smagghe et al., 2005). 20E induces a transcriptional regulatory cascade leading to PCD of larval tissues like intersegmental muscles (Lockshin \& Williams, 1965), salivary glands (Jiang et al., 2000), silk glands (Terashima et al., 2000) and larval midgut (Lee et al., 2002; Nishiura, 2002).

Ecdysone regulated gene cascade during salivary gland cell death in Drosophila melanogaster has been studied in detail (Baehrecke, 2000). High ecdysone levels at the end of the last larval instar triggers PCD through heterodimeric receptor complex, EcR and ultraspiracle (USP), which induces the expression of early genes E93, the zinc finger transcription factor Broad complex (BR-C) and the ETS family of transcription factors, E74 and E75. The products of early genes trigger the expression of late or effector genes including Drosophila hormone receptors (DHR3), DHR39 ( $\beta$ FTZ-F1) and E78, which perform the metamor- phic changes like PCD of larval tissues or growth and differentiation of imaginal cells into adult tissues (Jiang et al., 1997).

The PCD of larval midgut in different species, including Drosophila melanogaster (Denton et al., 2009, 2010), Aedes aegypti (Parthasarathy \& Palli, 2007), Heliothis virescens (Tettamanti et al., 2007) and Bombyx mori (Goncu \& Parlak, 2011; Franzetti et al., 2012) has been studied in detail. Previous studies demonstrate the existence of different mechanisms of PCD in larval midgut degeneration in different species of insects. For example, degeneration of the larval midgut in Drosophila depends on autophagy, not caspase-dependent apoptosis (Denton et al., 2009, 2010; Santhanam et al., 2014); however both apoptosis and autophagy are involved in larval midgut degeneration in lepidopteran insect species, such as Galleria mellonella (Khoa \& Takeda, 2012) and Bombyx mori (Goncu \& Parlak, 2011; Franzetti et al., 2012, 2015).

Previous studies on the remodeling of the midguts of different insect species also focused on the expression patterns of genes under ecdysone control. We previously reported that EcR B1 and USP-1 mRNAs are expressed in mature larval midgut cells during PCD. Decrease in E75A and probably BR-C Z4 mRNA levels in mature midgut 
cells appear to provide a signal for the initiation of this process. E74B, E75B and BR-C Z2 seem to be early response genes, which are involved in the preparatory phase of cell death. In addition, it is likely that $\beta F T Z-F 1$, E74A and BR-C Z1 are associated with execution phase of cell death (Goncu et al., 2016). Nishiura et al. (2005) reported that the expressions of AHR3, AaE75B, AaEcR-B and AaUSP-A change in Aedes aegypti during the 4th larval instar and that the juvenile hormone analogue methoprene alters the expressions. Application of juvenile hormone analogue prevents PCD in the epithelium of the larval midgut of Tribolium castenum and this inhibition is related to blocking the expression of EcR A, EcR B, Broad, E74, E75A and E75B (Parthasarathy \& Palli, 2009). Studies of PCD in the larval midgut of Drosophila reveal that some of the early genes like Broad and E93 are quite important in this process (Lee et al., 2002). We previously showed that fenoxycarb mimics juvenile hormone action and influences midgut remodeling in $B$. mori by interfering with the expression of EcR B1 in the 20E signal pathway (Goncu \& Parlak, 2012).

There is no information on the mechanism by which juvenile hormone affects the ecdysone-regulated gene cascade during PCD of the larval midgut in Bombyx mori. Various juvenile hormone analogues are used to clarify the anti-metamorphic effects of JH related to the ecdysone regulatory pathway. Kamimura \& Kiuchi (1998) showed that fenoxycarb, one of the most potent juvenile hormone analogues, mimics the action of $\mathrm{JH}$ actions in $B$. mori. When $1 \mathrm{ng}$ fenoxycarb is applied at the beginning of the $5^{\text {th }}$ larval instar, feeding during this stage is prolonged due to a delay in the secretion of ecdysone from the prothoracic gland, but this application does not prevent larval-pupal metamorphosis (Kamimura \& Kiuchi, 1998). This dose of fenoxycarb was therefore used in the present study. We explain differences in the pattern of gene expressions during a normal (control) and prolonged (after fenoxycarb treatment) feeding period as a response to delayed ecdysone secretion. However, differences in gene expression during the prepupal and early pupal stages indicate that fenoxycarb may also have a direct effect on the expression of genes belonging to the ecdysone-regulated gene cascade.

\section{MATERIAL AND METHODS}

\section{Insect rearing, fenoxycarb application and staging}

Japanese $\times$ Chinese hybrid races of the silkworm, Bombyx mori, were reared on fresh mulberry leaves at $25 \pm 1^{\circ} \mathrm{C}, 75-80 \%$ relative humidity and under a $12 \mathrm{~L}: 12 \mathrm{D}$ photoperiod. The last instar larvae were topically treated with $1 \mathrm{ng} / 10 \mu \mathrm{L}$ of fenoxycarb dissolved in acetone immediately after the fourth larval ecdysis (day 0). Experiments were carried out every $12 \mathrm{~h}$ from day 7 larvae of the $5^{\text {th }}$ instar up to $12 \mathrm{~h}$ after pupation. We divided the experimental period into four stages based on morphological features. Feeding stage: In the control group, the $5^{\text {th }}$ instar larvae fed actively up to the end of day 7 . They showed normal larval life stage characteristics and their midgut was healthy. The feeding stage of $5^{\text {th }}$ instar larvae treated with fenoxycarb is prolonged and continued up to the end of day 10. Early prepupal stage: Control group of insects stopped feeding at the beginning of day 8 , when gut purge occurred. Larvae exhibited wandering behaviour, then spinning began on this day. Larvae were completely surrounded by cocoons on day 9 of the $5^{\text {th }}$ larval instar but spinning continued. Fenoxycarb treated insects stopped feeding in the second half of day 10 and then began to spin a cocoon. Late prepupal stage: It lasted during day 10 of the $5^{\text {th }}$ larval instar in control group insects and day 13 of the $5^{\text {th }}$ larval instar in fenoxycarb treated insects. Spinning activity had almost finished in the second half of this stage and larvae were quiescent (Pharate pupae). Early pupal stage: It lasted from larval-pupal ecdysis to $12 \mathrm{~h}$ after pupation.

\section{Preparation of cross sections of midguts for staining}

Midgut tissues were isolated every $12 \mathrm{~h}$ from day 7 up to $12 \mathrm{~h}$ after pupation. They were fixed in Bouin's solution for 5-6 h at $4^{\circ} \mathrm{C}$. Fixed tissues were dehydrated by putting them in a graded series of ethanol and then processed for embedding in paraffin wax. Five micrometer-thick sections were used for the morphological evaluation. Sections were stained with hematoxylin and eosin (H\&E) using a routine protocol and examined under a Leica DM3000 microscope.

\section{TUNEL assay}

DNA fragmentation in apoptotic cells was identified by using the In Situ Cell Death Detection Kit, Fluorescein (Roche, Germany). Nuclear staining was done using DAPI (4 6-diamidino-2 phenyl indol, Invitrogen, USA). Sections were examined under a Leica DM3000 microscope and photographed using a digital camera. Images were analyzed using image J analysis (http://imagej.nih.gov/ij/).

\section{Acid phosphatase assay}

The activity of acid phosphatase (AP) in the midguts and yellow bodies was measured using paranitrophenol phosphate (Sigma, St Louis, MO, USA) as a substrate according to Bergmeyer et al. (1974). The measurements were repeated on three different series of animals. The optical density of the liberated paranitrophenol was read at $405 \mathrm{~nm}$ using a spectrophotometer.

\section{Western blotting}

Midguts were homogenized in $100 \mathrm{mM}$ mannitol, $10 \mathrm{mM}$ HEPES-Tris $\mathrm{pH}$ 7.2, containing a protease inhibitor cocktail (Complete Mini, Roche) for cleaved caspase 3 and $20 \mathrm{mM}$ HEPES (pH 7.5), $250 \mathrm{mM}$ sucrose, $1 \mathrm{mM}$ EGTA, $1 \mathrm{~m}$ M EDTA, $100 \mathrm{mM} \mathrm{KCl}, 1$ mM DTT, 0.1 mM PMSF, 2 ug/ml Pepstatin, containing the protease inhibitor cocktail (Complete Mini, Roche) for cytochrome $\mathrm{c}$. The homogenates were centrifuged at 15,000 $\mathrm{g}$ for $15 \mathrm{~min}$ at $+4^{\circ} \mathrm{C}$. Total protein concentration was determined by using a bicinchoninic acid (BCA) protein assay kit (Pierce) employed according to manufacturer's instructions. Twenty micrograms of total proteins were separated by $15 \%$ sodium dodecylsulfate(SDS)-polyacrylamide gel electrophoresis in a gel running buffer (25 mM Tris, $192 \mathrm{mM}$ glycine, $0.1 \% \mathrm{SDS}, \mathrm{pH}$ 8.3) using a Bio-Rad vertical electrophoresis system, then, the proteins were electrotransferred onto a nitrocellulose membrane (Pierce, 88018) using a Bio-Rad Transblot cell. Membranes were placed in blocking solution containing $50 \mathrm{mM}$ Tris-HCl, $\mathrm{pH} 7.5$, $150 \mathrm{mM} \mathrm{NaCl}, 1 \mathrm{~m} \mathrm{M}$ EDTA and $0.1 \%$ Tween 20 (TBST) containing $1 \%$ bovine serum albumin and $5 \%$ dried non-fat milk, overnight at $4^{\circ} \mathrm{C}$. They were then incubated with a 1:1000 dilution of 6B7 anti-cleaved caspase 3 antibody (ASP 175, Cell Signaling Technology) and cytochrome c antibody (4272, Cell Signaling Technology) and beta-actin (4967, Cell Signaling Technology) overnight at $4{ }^{\circ} \mathrm{C}$ followed by $2 \mathrm{~h}$ incubation with a $1: 1000 \mathrm{di}-$ lution of horseradish peroxidase conjugated secondary antibody (Cell Signaling Technology, 7074). Detection was performed by chemiluminescence (Pierce, ECL western blotting substrate, 
32106) according to manufacturer's instructions. The results were analyzed using a Chemidoc (Biorad) imaging system.

\section{RNA isolation and cDNA synthesis}

The midguts isolated from day 7 to day 9 from control and day 7 to day 13 from fenoxycarb treated insects were prepared for removing stem cells from mature midguts according to Hakim et al. (2009). Midgut stem cells lie loosely associated among the bases of the epithelial cells, therefore, they can be effectively separated by using this method (Hakim et al., 2009; Goncu et al., 2016). Midgut lumen contents, which contained only a yellow body from day 10 of the $5^{\text {th }}$ instar up to $12 \mathrm{~h}$ after pupation in control and day 13 of the $5^{\text {th }}$ instar up to $12 \mathrm{~h}$ after pupation in the fenoxycarb treated insects, were pooled and all mature midgut cell samples collected in Tripure Isolation Reagent (Roche) for every $12 \mathrm{~h}$. Samples were homogenized in the Tripure reagent and total RNA was isolated according to manufacturer's instructions. Concentration of total RNA and purity were evaluated using a Nanodrop UV/VIS spectrophotometer. cDNA synthesis by reverse transcription was performed using $1 \mu \mathrm{g}$ of RNA and a High Fidelity cDNA synthesis kit (Roche) in a $20 \mu 1$ reaction volume.

\section{Quantitative real-time reverse transcriptase polymerase chain reaction (qRT-PCR)}

Relative expressions of particular genes were evaluated using qRT-PCR and a lightcycler 480 real time PCR detection systems (Roche) as previously described (Gibson et al., 1996). qRT-PCR was performed in a total reaction volume of $10 \mu 1$ containing 2 $\mu \mathrm{l}$ of cDNA, $0.5 \mu \mathrm{l}$ each of forward and reverse sequence spe- cific primers, $2.7 \mu 1 \mathrm{H}_{2} \mathrm{O}, 0.2 \mu 1$ Tprobe and $5 \mu 1$ enzyme. PCR conditions were $95^{\circ} \mathrm{C}$ for $10 \mathrm{~min}$ followed by 45 cycles of $95^{\circ} \mathrm{C}$ for $10 \mathrm{~s}, 60^{\circ} \mathrm{C}$ for $30 \mathrm{~s}, 72^{\circ} \mathrm{C}$ for $1 \mathrm{~s}, 40^{\circ} \mathrm{C}$ for $30 \mathrm{~s}$. The primers used for the PCR were derived from sequences of Bombyx genes and the references are listed in Table 1. Primer probe design was performed using clustal $\mathrm{W}$ align and oligo7 software. Specificity of the primers and probes was determined using the blast program. Bombyx mori Actin 3 was used as an endogenous control. Mean and standard errors were obtained from the averages of three independent sample sets. For each gene, we determined gene expressions at different developmental stages using the One-way Analysis of Variance (ANOVA) in SPSS statistics software (IBM).

\section{RESULTS}

\section{Feeding and early prepupal stages}

During the feeding period of the control (Fig. 1A) and the prolonged feeding period of the treated group (Fig. 1B), midguts consisted of healthy columnar epithelial cells, goblet cells and a few stem cells located in the basal region of the epithelium . Early prepupal stage started when feeding ceased at the end of day 7 in control and in the second half of day 10 in treated insects. First morphological signs of apoptosis, especially cell shrinkage and nuclear and cytoplasmic condensation in the columnar and goblet cells, were observed in both groups during the early prepupal stage (Fig. 1A, B). TUNEL staining revealed DNA frag-

Table 1. Forward and reverse primers used in qRT-PCR.

\begin{tabular}{|c|c|c|c|}
\hline Gene & Forward and reverse primers & Accession no. & upl prob no. \\
\hline B. mori actin 3 & $\begin{array}{l}\text { 5'-GCTCCCTCGAGAAGTCTTACG-3' } \\
\text { 5'-CTGGGCAACGGAATCTTTC-3' }\end{array}$ & U49854 & 9 \\
\hline EcR-A & $\begin{array}{l}\text { 5'-CATCCGGTCAACGGACAC-3' } \\
\text { 5'-ACCGTAGCTGCCTGAGGATA-3' }\end{array}$ & D87118 & 141 \\
\hline EcR-B1 & $\begin{array}{l}\text { 5'-ACTTGGCAGTCGGATGAAG-3' } \\
\text { 5'-CGTCATCTCCGTGATCTGG-3' }\end{array}$ & L35266 & 153 \\
\hline USP-1 & $\begin{array}{l}\text { 5'-TCAAATAGGCAACAAACAGATAGCCGCTC-3' } \\
\text { 5'-CAGGAACTCCATAGACCG-3' }\end{array}$ & U06073 & 150 \\
\hline USP-2 & $\begin{array}{l}\text { 5'-CAGTGTCACATGTAGAGTGCAAAGA-3' } \\
\text { 5'-CCACTTTCATAGAACAGTTCAGTTGC-3' }\end{array}$ & \multicolumn{2}{|c|}{$\begin{array}{l}\text { AB182582 FAM-GTTCAACGACCTTGTTGCTGACAGGTTC-Tamra } \\
\text { Taqman probe }\end{array}$} \\
\hline E74A & $\begin{array}{l}\text { 5'-CCACCTATCGAGATAAAGCAAGA-3' } \\
\text { 5'-CTTGCAGTTGACTGCTGGAC-3' }\end{array}$ & Q1KLS0 & 141 \\
\hline E74B & $\begin{array}{l}\text { 5'-ACCCGAGTGACTACGTGAGG-3' } \\
\text { 5'-CGGAGTCTGTGCCTGAGTCT-3' }\end{array}$ & Q1KLR9 & 95 \\
\hline E75A & $\begin{array}{l}\text { 5'-TCGGTCGAGCTTGAGTGAG-3' } \\
\text { 5'-GATGAAGGTCGCTTGTCTCG-3' }\end{array}$ & AB024904 & 59 \\
\hline E75B & $\begin{array}{l}\text { 5'-GGACAGCTCTCAAAGACGTGA-3' } \\
\text { 5'-CGCACCATTCACTACACTCG-3' }\end{array}$ & AB024905 & 91 \\
\hline BR-C Z1 & $\begin{array}{l}\text { 5'-TCTGCAGAGTCCTCTCGCTTC-3' } \\
\text { 5'-TACACGCGCTGGCAAATG-3' }\end{array}$ & AB166725 & 36 \\
\hline BR-C Z2 & $\begin{array}{l}\text { 5'-TCTGCAGAGTCCTCTCGCTTC-3' } \\
\text { 5'-GTGTATATGTGCGTCATCAGGGA-3' }\end{array}$ & AB166726 & 99 \\
\hline BR-C Z4 & $\begin{array}{l}\text { 5'-TCTGCAGAGTCCTCTCGCTTC-3' } \\
\text { 5'-TCTTGTGGTTGTTGAGCGAGTT-3' }\end{array}$ & AB166727 & 56 \\
\hline$\beta F T Z-F 1$ & $\begin{array}{l}\text { 5'-TTCCGCAAGTATCATCATTGAC-3' } \\
\text { 5'-CTTGTCGTGAGTTGGTGGTG-3' }\end{array}$ & D10953 & 141 \\
\hline BHR3 & $\begin{array}{l}\text { 5'-GGGATGCAAAGGATTCTTCA-3' } \\
\text { 5'-GCGAGGACACTGGTAGTTCAC-3' }\end{array}$ & AB024902 & 9 \\
\hline BmATG8 & $\begin{array}{l}\text { 5'-TCCGGAAACGTATTCACCTG-3' } \\
\text { 5'-GGGTGGAATGACATTGTTCAC-3' }\end{array}$ & FJ416330 & 124 \\
\hline BmATG12 & $\begin{array}{l}\text { 5'-CCTGTATGTGAATCAGACTTTTTGC-3' } \\
\text { 5'-CCGAAGCACTCATAAAGATTCC-3' }\end{array}$ & FJ416329 & 9 \\
\hline
\end{tabular}


A
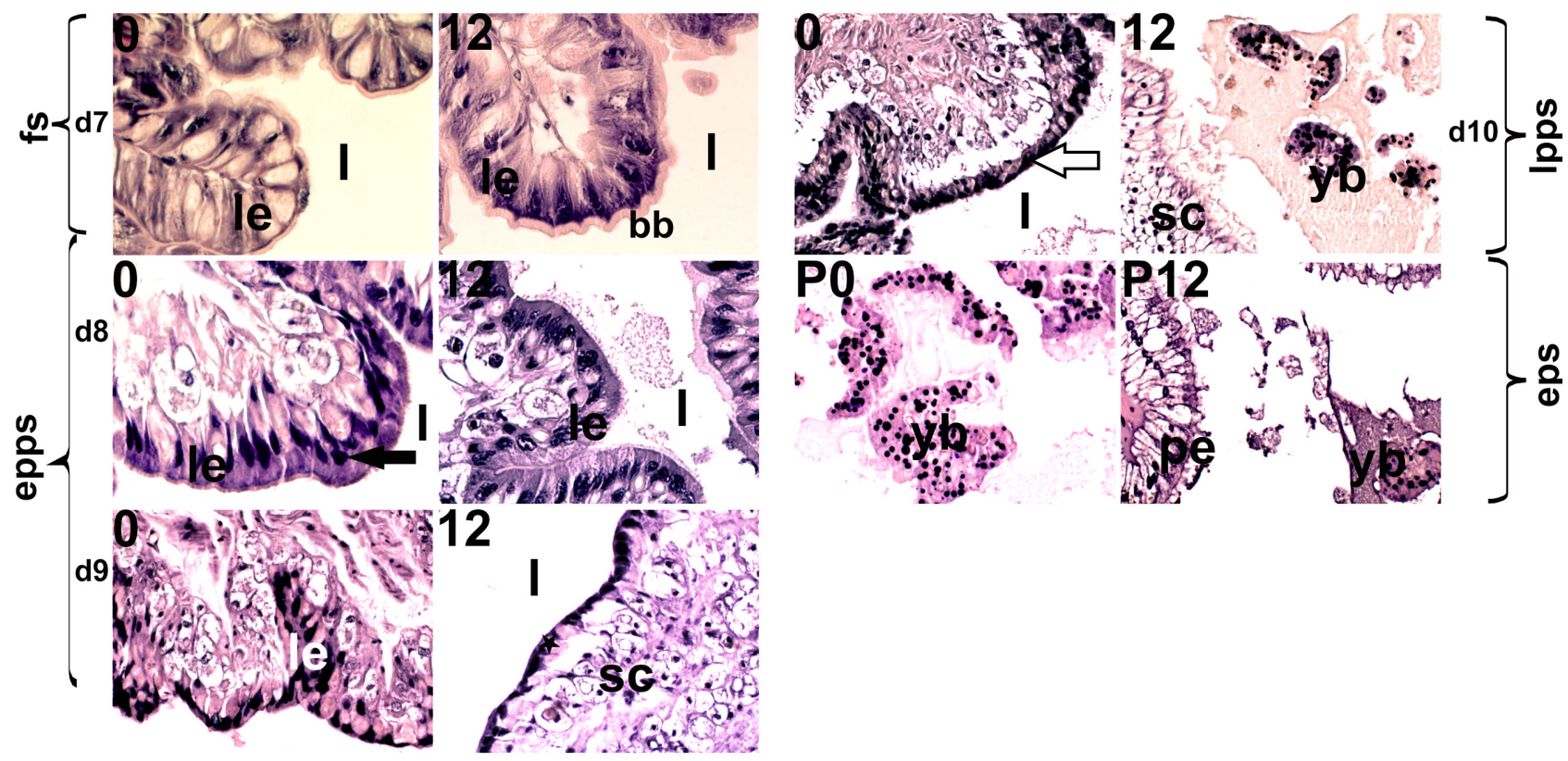

B
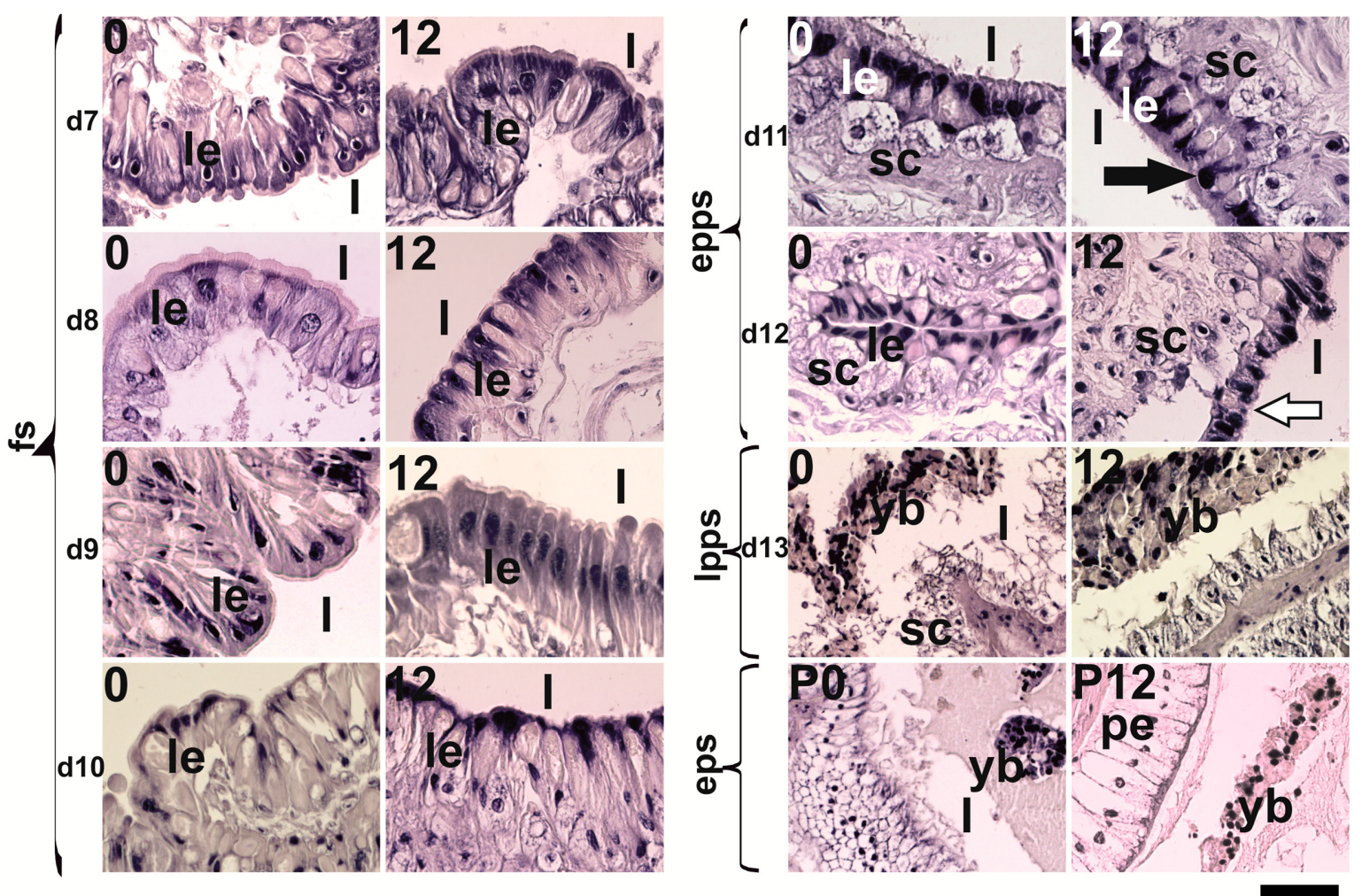

Fig. 1. Histology of Bombyx mori midgut in the control (panel A) and fenoxycarb treated insects recorded (panel $\mathrm{B}$ ) at 0 and $12 \mathrm{~h}$ of indicated days (d7-d13) of the last larval instar (fs - feeding stage; epps - early prepupal stage; lpps - late prepupal stage) and at 0 and $12 \mathrm{~h}$ after pupal ecdysis (eps - early pupal stage). Additional abbreviations: le - healthy larval epithelium; bb - brush border membrane; I - gut lumen; sc - enlarged stem cells (black arrow indicates degenerating epithelium with nuclear and cytoplasmic condensation and white arrow shows the remains of epithelium in the gut lumen); yb - yellow body; pe - pupal epithelium. Scale bar $=50 \mu \mathrm{m}$.

mentation, which is an indicator of apoptosis (Fig. 2A). In the midguts of the control group, only a few TUNEL positive cells $(1 \%)$ were recorded during the early prepupal stage (Fig. 2B). Cytosolic cytochrome c and cleaved caspase 3, which are critical indicators of apoptosis, were revealed by western blotting (Fig. 3A, B) and their relative abundances calculated using Image Lab software (Biorad) Fig. 3C, D). In the untreated insects, the most abundant 


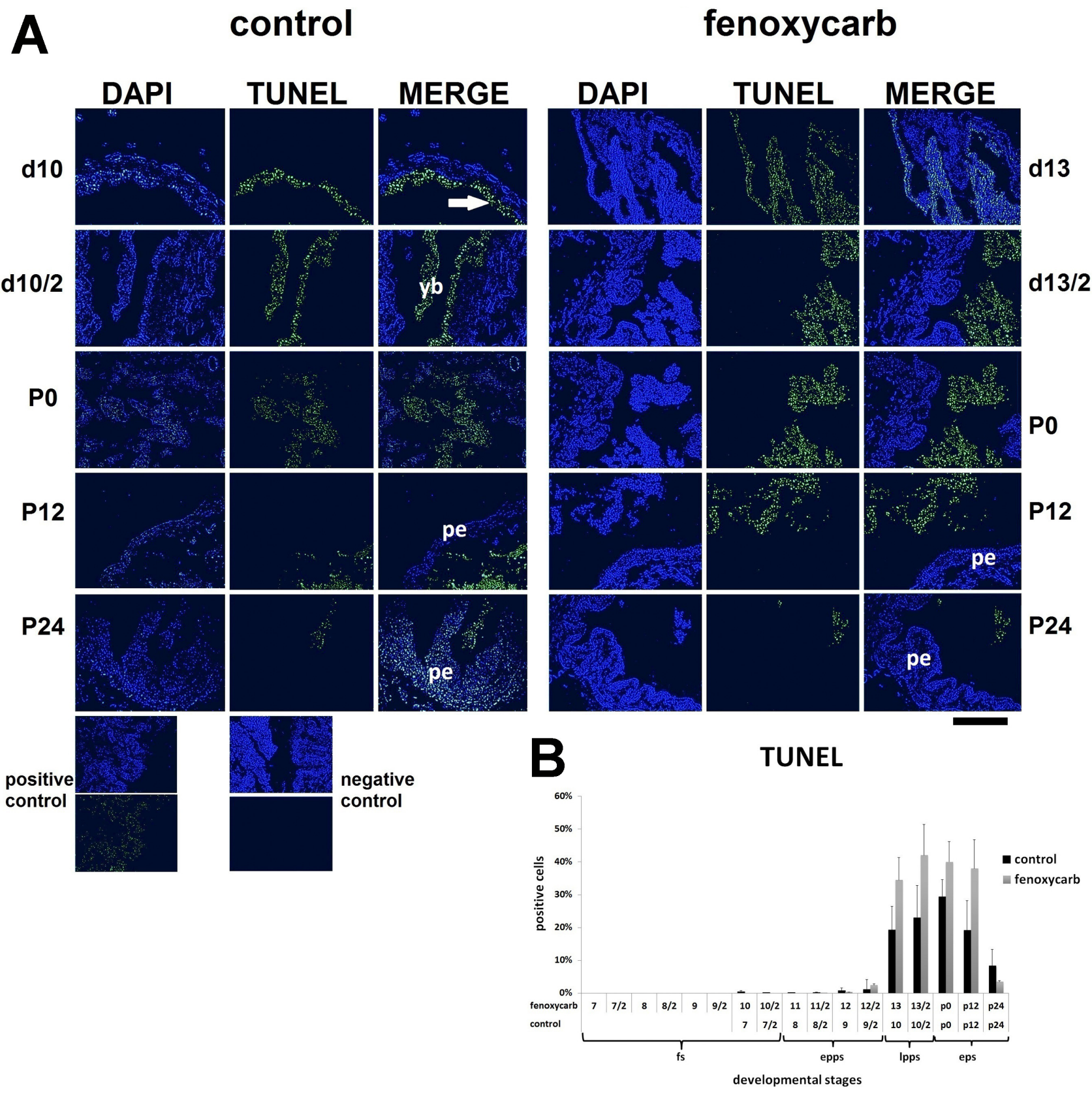

Fig. 2. Apoptosis specific DNA fragmentation detected in the midguts of control and fenoxycarb treated insects. White arrow indicates positive signals located in the detached larval epithelium on day 10 (d10). Positively stained nuclei on the second half of day 10 (d10/2), at pupation (p0) $12 \mathrm{~h}$ after pupation (p12), and $24 \mathrm{~h}$ after pupation (p24). Positive signals in midguts of fenoxycarb treated insects recorded on day 13 of the $5^{\text {th }}$ instar, with a sample of DNase treated larvae recorded on day 7 as a positive control, p0 samples were used as a negative control. Additional abbreviations: $y b-$ yellow body; pe - pupal epithelium. Scale bar $=150 \mu \mathrm{m}(\mathrm{A})$. Intensity of the TUNEL positive signals (B). Because the prolonged feeding period of the fenoxycarb treated insects, the $X$ axis of this graph indicates the developmental stages. (fs - feeding stage; epps - early prepupal stage; Ipps - late prepupal phase; eps - early pupal stage.

cytosolic cytochrome c was detected only in the first half of day 7 (Fig. 3A) and its calculated relative abundance was $57.09 \%$ (Fig. 3C). Positive signals were detected every 12 h on day 8 (Fig. 3A) and the relative abundances were $18 \%$ and $11 \%$, respectively (Fig. 3C). However cleaved caspase 3 was not recorded in the feeding and early prepupal stages (Fig. 3A). The expression of the ATG8 and ATG 12 genes, and enzyme activity of acid phosphatase were used as indicators of autophagy (Fig. 4). Interestingly, despite the low levels of ATG 8 during the feeding and early prepupal stages of the control group (Fig. 4A); the highest ATG 12
mRNA level was detected on day 7 (Fig. 4B). In addition to this, moderate acid phosphatase activity was detected during the feeding and early prepupal stages (Fig. 4C). In the midguts of the fenoxycarb treated insects, a few TUNEL positive cells $(2 \%)$ were first detected in the second half of day 12 (Fig. 2B). The highest abundance of cytosolic cytochrome c $(76 \%)$ was recorded in the second half of day 9. Positive signals were detected every $12 \mathrm{~h}$ from day $10 \mathrm{up}$ to the first half of day 11 with relative abundances of $22 \%$, $16 \%$ and $10 \%$, respectively (Fig. 3B, D). Similar to the control, cleaved caspase 3 was not determined during the 

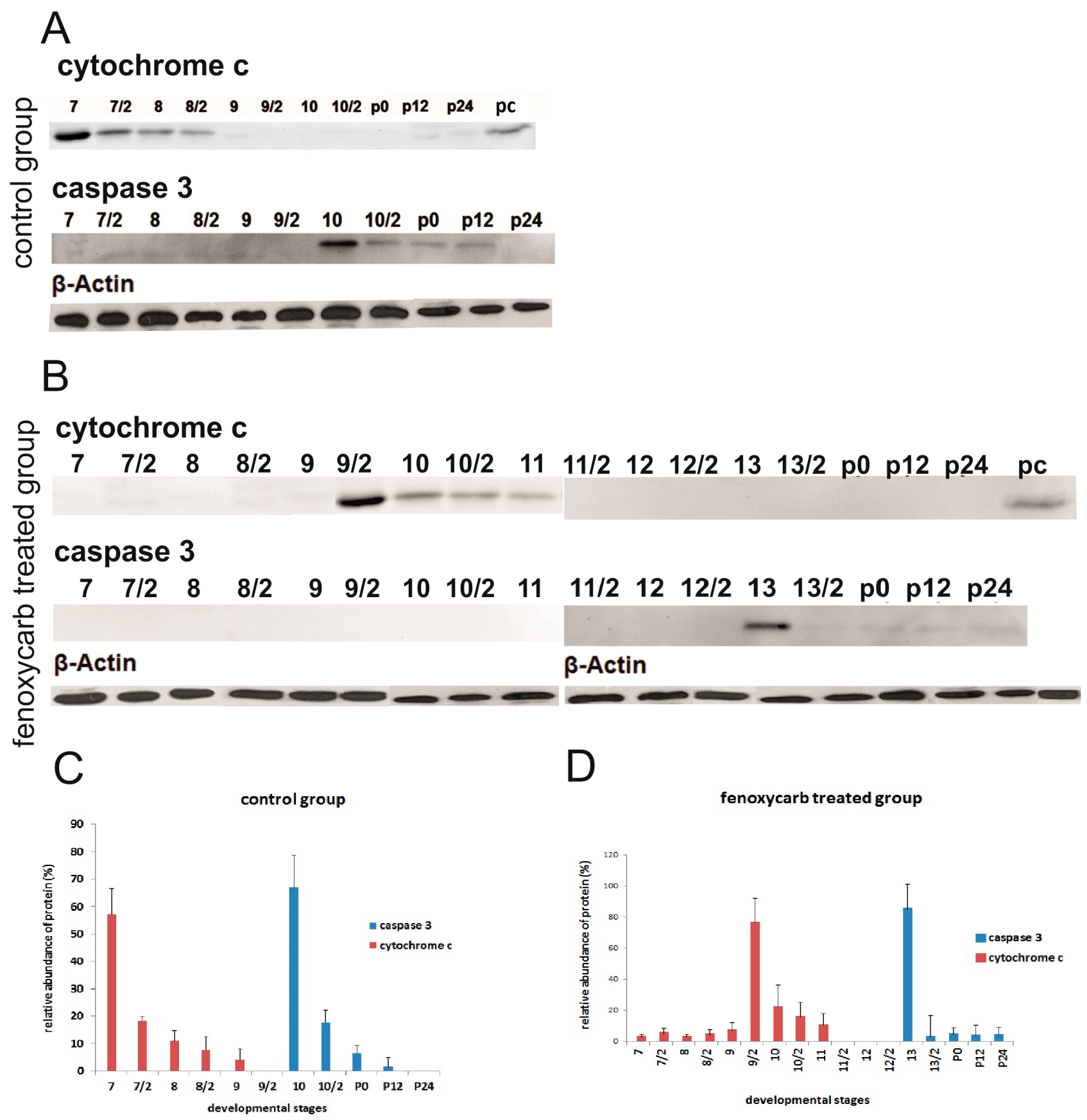

Fig. 3. Western blot analysis of cytosolic cytochrome $c$ and cleaved caspase 3 in the control $(A)$ and fenoxycarb treated insects (B) on the days indicated (d7-d13) during the last larval instar and after pupation ( $0,0 \mathrm{~h} ; \mathrm{p} 12,12 \mathrm{~h} ; \mathrm{p} 24,24 \mathrm{~h}$ ). Mitochondrial fraction was used as a positive control (pc) Beta-actin was used as a loading control. Relative abundance was calculated using Image Lab software (Biorad) $(C, D)$. Mean \pm S.D. for the three independent experiments are shown.

extended feeding stage and early prepupal stages (Fig. 3B). Moderate ATG8 mRNA levels were detected up to day 12 of the $5^{\text {th }}$ larval instar, and then decreased to low levels at the end of the early prepupal stage (Fig. 4A) however, the highest ATG 12 levels were detected during the extended feeding period of treated insects up to the second half of day 9 after which it decreased up to the end of the early prepupal stage (Fig. 4B). Low acid phosphatase activities gradually increased during the prolonged feeding stage and remained at a moderate level up to the second half of day 12. The highest enzyme activity was detected at the end of early prepupal stage (Fig. 4C).
The expression patterns of ecdysone receptors and ecdysone related transcription factors were analyzed using qRTPCR (Fig. 5). Due to the prolonging of the feeding period in fenoxycarb treated insects, plots depict the expression of ecdysone receptors and ecdysone-related transcription factors in particular developmental stages. The expression of EcR A in the midguts of the control group of insects was high during the actively feeding stage on day 7 of the $5^{\text {th }}$ larval instar after which it abruptly decreased when feeding ceased (Fig. 5a). Unlike EcR A, expression levels of EcR B1 peaked on day 7 and day 8 of the last larval instar and was nearly 80 -fold higher than levels of EcR A mRNA 
A

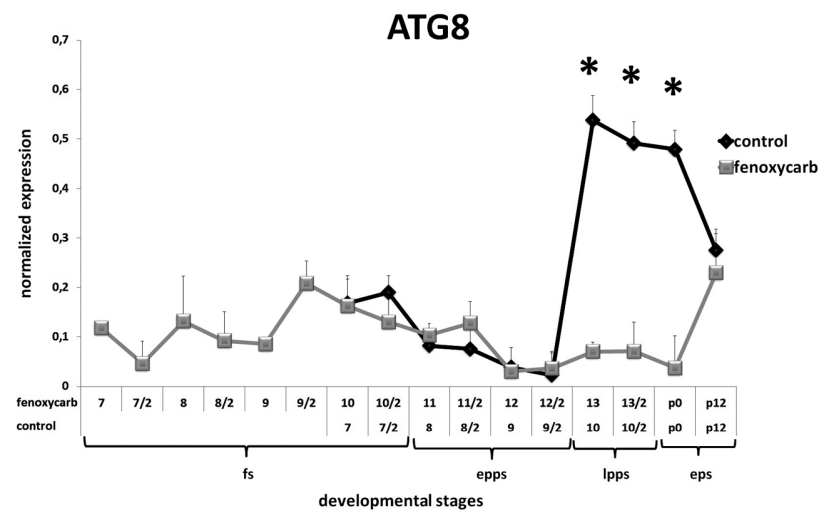

B

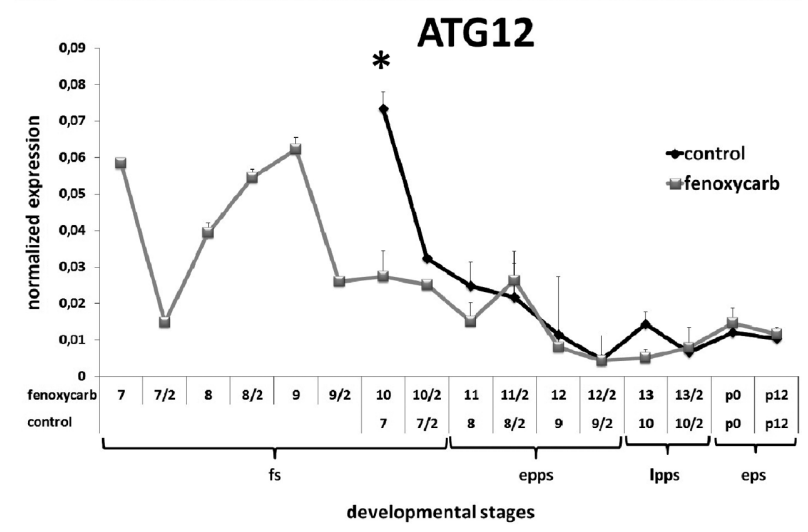

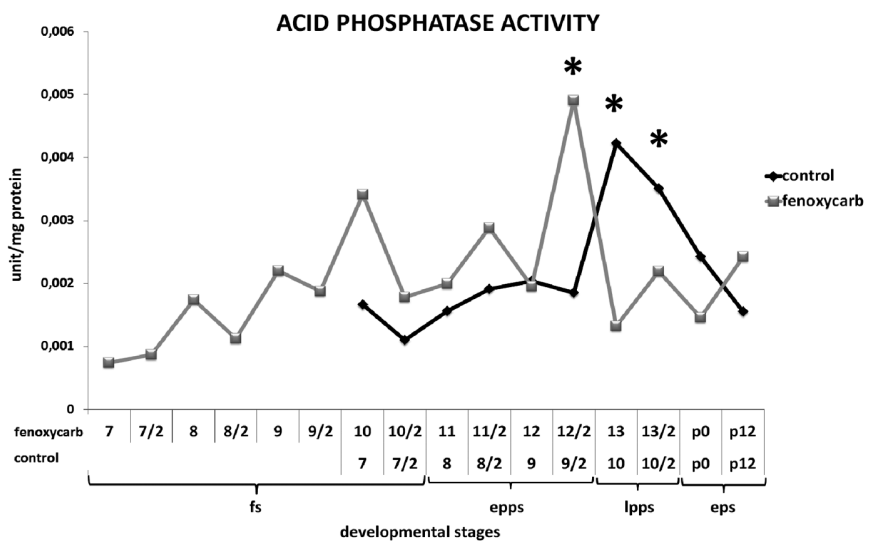

Fig. 4. Expression patterns of the autophagy related genes ATG8 (A), ATG 12 (B) and acid phosphatase activity (C) in control and fenoxycarb treated insects. Analyzes were performed every $12 \mathrm{~h}$ of indicated days (d7-d13) of the last larval instar (fs - feeding stage; epps - early prepupal stage; lpps - late prepupal stage) and at 0 and $12 \mathrm{~h}$ after pupal ecdysis (eps - early pupal stage). Because of the prolonged feeding period of the fenoxycarb treated insects, plots of the expression of autophagy related genes and enzyme activity are based on the developmental stages. Expression levels of the genes were normalized using Bombyx mori Actin 3 RNA as a standard. Mean \pm S.D. are for the three independent experiments. Asterisks indicate significant differences in expression and enzyme activity $(P \leq$ 0.05 ) using one-way ANOVA.

(Fig. 5b). The expression profiles of USP 1 (Fig. 5c) were similar to those of EcR B1. Moderate USP2 mRNA levels on day 7 and day 8 decreased to very low levels on day 9 of the $5^{\text {th }}$ larval instar when it was in the second half of the early prepupal stage (Fig. 5d). The mRNAs for both E74 isoforms showed similar expression profiles but the relative abundance of E74B was 10-fold higher than that of E74A. The average mRNA levels up to day 9 of the $5^{\text {th }}$ larval instar decreased to very low levels by the end of early prepupal stage (Fig. 5e, f). A relatively high expression rate of the other early transcription factor, E75A, was recorded during the feeding stage but, after feeding ceased, its expression was abruptly downregulated on day 8 and then remained at a very low level (Fig. $5 \mathrm{~g}$ ). There was a transient peak in the low expression level of E75B just before the termination of feeding on day 8 of the $5^{\text {th }}$ larval instar and a decline in expression thereafter (Fig. 5h). BR$\mathrm{C}$ isoforms have different expression patterns. The high expression of BR-C Z1 in the second half of day 7 gradually decreased up to day 10 of the $5^{\text {th }}$ larval instar (Fig. 6a). Among the BR-C isoforms, the most abundant was BR-C $\mathrm{Z} 2$ with moderate levels detected during the feeding stage, which peaked on day 9 (Fig. 6b). The highest BR-C Z4 mRNA level was recorded when the larvae were actively feeding and after feeding ceased its expression sharply decreased. At the beginning of the early prepupal stage, the BR-C Z4 transcripts began to increase again with a small peak on day 9 of the $5^{\text {th }}$ larval instar, then declined again to undetectable levels (Fig. 6c). Moderate $\beta$ FTZ-F1 mRNA levels gradually decreased in a step-wise manner after the termination of feeding on day 8 of the $5^{\text {th }}$ larval instar up to day 10 in the late prepupal stage (Fig. 6d). Levels of BHR3 transcripts were undetectable during the feeding and early prepupal stages (Fig. 6e).

The expression of EcR A mRNA in midguts of fenoxycarb treated insects was relatively high during the extended feeding stage from day 7 to day 11 of the $5^{\text {th }}$ larval instar (Fig. 5a). However, EcR A transcripts in midguts of the control group of insects were almost three-fold higher than those in the fenoxycarb treated insects. After cessation of feeding, the expression of EcR A mRNA decreased to very low levels (Fig. 5a). Similar to EcR A, fenoxycarb treatment also reduced the expression of EcR B1 during the feeding stage and prevented the occurrence of peaks 

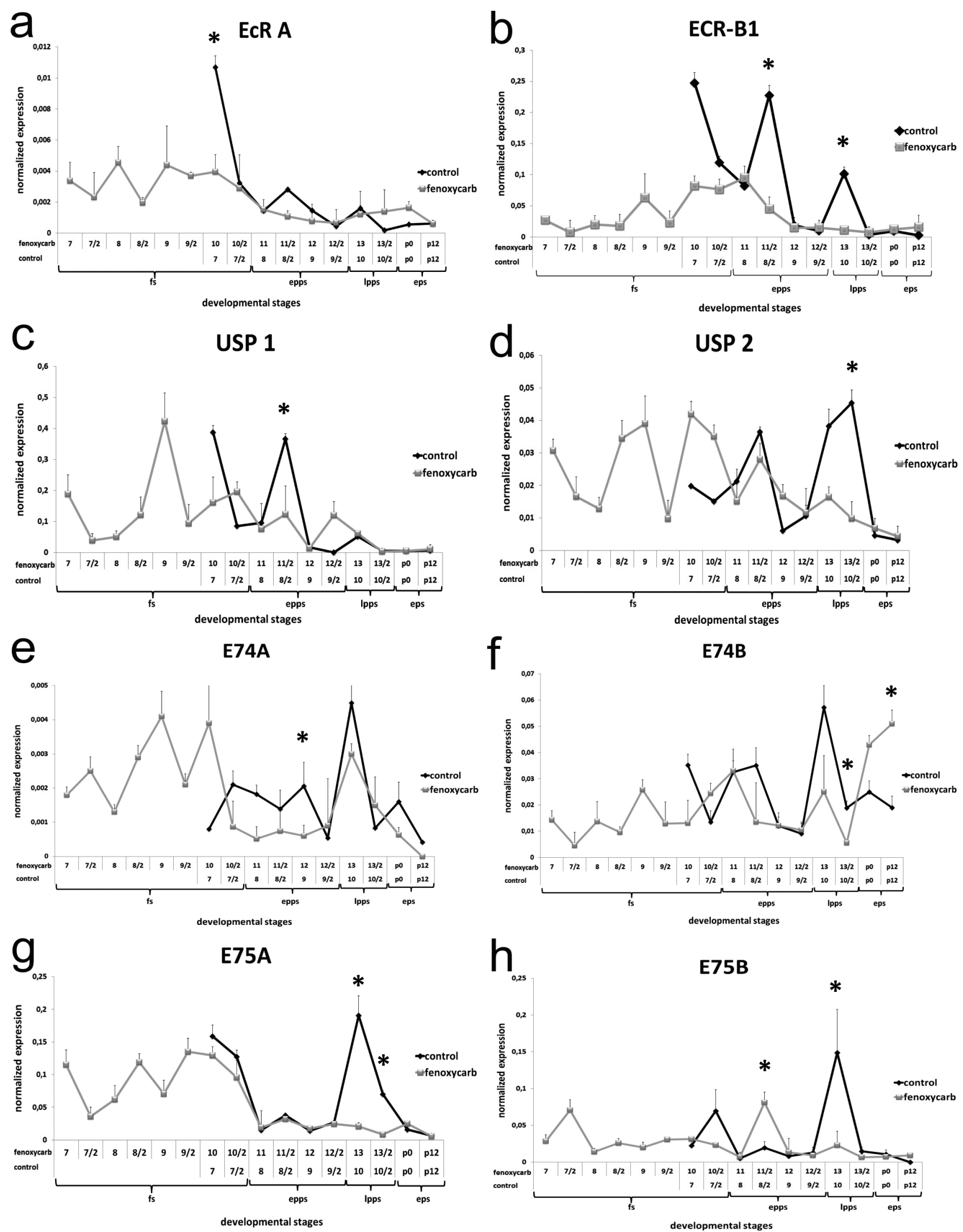

Fig. 5. Comparison of the ecdysone related gene expressions in larval midguts and yellow bodies of control and fenoxycarb treated insects. Sampling was carried out every $12 \mathrm{~h}$ of indicated days (d7-d13) of the last larval instar (fs - feeding stage; epps - early prepupal stage; Ipps - late prepupal stage) and at 0 and $12 \mathrm{~h}$ after pupal ecdysis (eps - early pupal stage). Because of the prolonged feeding period of fenoxycarb treated insects, plots of the expression of ecdysone receptors and ecdysone-related transcription factors are indicated for the different developmental stages. Ecdysone receptor A (EcR A) (a), ecdysone receptor B1 (EcR B1) (b), ultraspiracle 1 (USP1) (c), ultraspiracle 2 (USP2) (d), E74A (e), E74B (f), E75A (g), E75B (h). Expression levels of these genes were normalized using Bombyx mori Actin 3 RNA as a standard. Mean \pm S.D. for the three experiments are shown. Asterisks indicate significantly different expressions $(P \leq$ $0.05)$ based on a one-way ANOVA. 

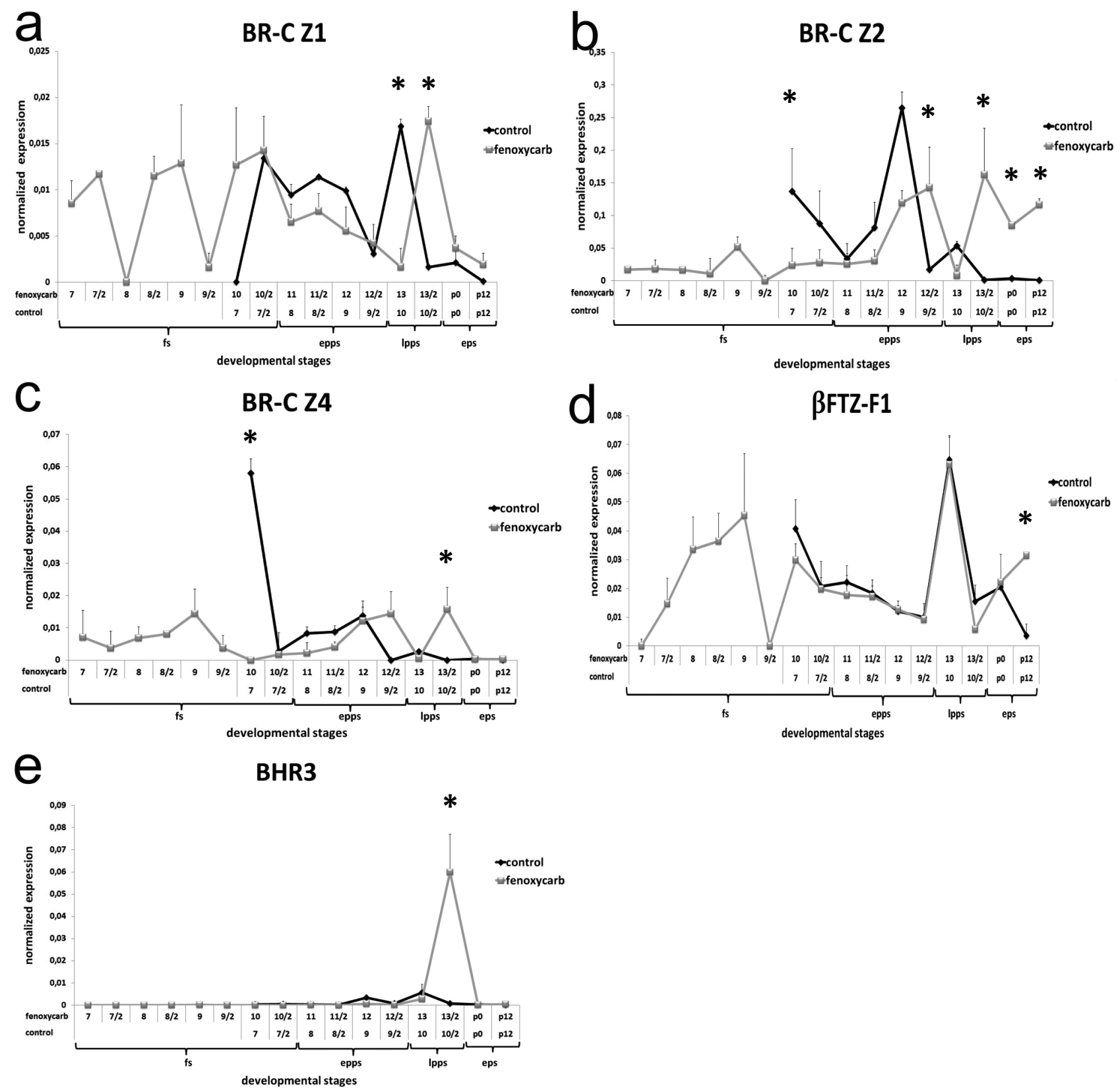

Fig. 6. Comparison of the ecdysone related gene expressions in larval midguts and yellow bodies of untreated and fenoxycarb treated insects (cont.) BR-C Z1 (a), BR-C Z2 (b), BR-C Z4 (c), BFTZ-F1 (d), BHR3 (e). Expression levels of these genes were normalized using Bombyx mori Actin 3 RNA as a standard. Mean \pm S.D. for the three experiments are shown. Asterisks indicate significantly different expressions $(P \leq 0.05)$ based on a one-way ANOVA.

in expression of EcR B1 during the early prepupal stage (Fig. 5b). The low expression levels of EcR B1 up to day 9 began to increase and remained almost steady up to day 12 of the $5^{\text {th }}$ larval instar (Fig. 5b). In contrast to ecdysone receptors, the highest USP 1 (Fig. 5c), USP 2 (Fig. 5d), E74A (Fig. 5e) and E75A (Fig. 5g) mRNA levels were detected in feeding insects in fenoxycarb treated group. Their expressions decreased after the termination of feeding and remained at low levels up to day 13 of the $5^{\text {th }}$ larval instar. In addition, USP1 (Fig. 5c) and E74A (Fig. 5e) mRNA levels were significantly lower than those in control group insects during the early prepupal stage $(p \leq 0.05)$. Levels of E74B mRNA fluctuated during the extended feeding period up to the second half of day 11 of the $5^{\text {th }}$ larval instar, and then decreased to low levels at the end of the early prepupal stage (Fig. 5f). Expression levels of E75B remained low up to the end of the early prepupal stage except for transient peaks in the second half of day 7 and second half of day 11 , respectively (Fig. $5 \mathrm{~h}$ ). The peak level in the second half of day 11 was four-fold higher than that found in the control. The high expression of BR-C Z1 detected during the extended feeding stage gradually decreased up to day 13 of the $5^{\text {th }}$ larval instar (Fig. 6a). In contrast to BR-C Z1, the expression of BR-C Z2 was maintained at very low levels up to day 11 and then gradually increased from the beginning of day 12 (Fig. 6b). It's mRNA level in the second half of day 12 was significantly higher than that recorded for the control group of insects. Fenoxycarb treat- 


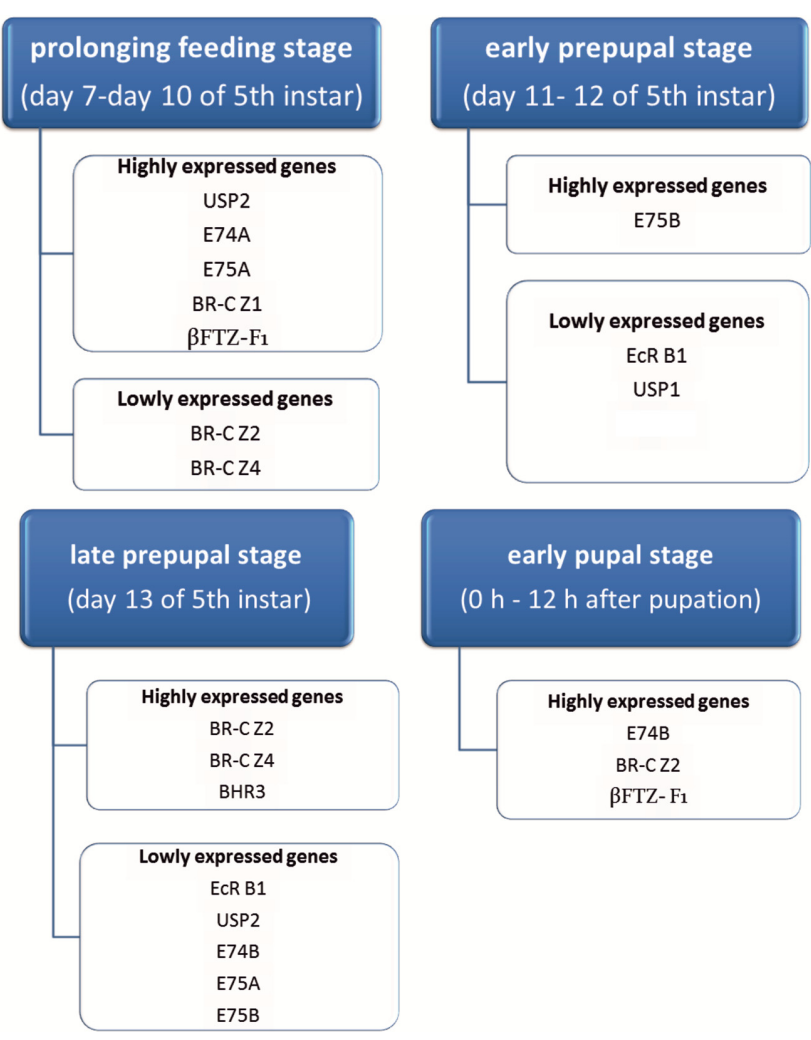

Fig. 7. Affected ecdysone receptors and ecdysone related transcription factors recorded at different developmental stages after treatment with fenoxycarb.

ment considerably reduced BR-C Z4 mRNA levels during the feeding stage. Low expression of BR-C Z4 during the early prepupal stage was similar to the expression profile of BR-C Z2 (Fig. 6c). Relatively high levels of $\beta$ FTZ-F1 mRNA were detected during the prolonged feeding period except for a transient decrease in the second half of day 9. After feeding ceased, its expression gradually decreased up to day 13 of the $5^{\text {th }}$ larval instar (Fig. 6d). Similar to the control, BHR3 transcripts were not detected in the midguts during the feeding and early prepupal stages of this group (Fig. 6e).

\section{Late prepupal stage}

In both groups degenerating larval epithelium separates locally from the basal lamina with increasing proliferation rate of stem cells and was the so called the yellow body, which is pushed into the midgut lumen during the late prepupal period (Fig. 1). In the midguts isolated from the control group, TUNEL positive cells rapidly increased (19.4\%) on day 10 of the $5^{\text {th }}$ larval instar and were also found in the yellow body (Fig. 2). Cleaved caspase 3, indicative of the onset of the execution phase, was determined for the first time during the late prepupal period and the highest relative abundance of it $(67 \%)$ occurred on this day (Fig. $3 \mathrm{~A}, \mathrm{C})$. The expression of the ATG 8 gene increased and peaked during the late prepupal stage (Fig. 4A) however, ATG 12 levels were very low at that time (Fig. 4B). Similar to ATG8, the highest acid phosphatase activities were detected during the late prepupal stage (Fig. 4C).
In the midguts isolated from fenoxycarb treated insects, the number of TUNEL positive cells sharply increased on day 13 of the $5^{\text {th }}$ larval instar $(32.3 \%)$ during the late prepupal stage and peaked (42.6\%) just before pupation (Fig. 2). The highest cleaved caspase 3 was detected at the beginning of the late prepupal stage and its relative abundance was $86 \%$ (Fig. 3B, D). ATG8 mRNA levels were significantly lower than those determined in the midguts of the control group $(\mathrm{p} \leq 0,05)$ (Fig. 4A) but the levels of ATG 12 mRNA were similar to those recorded in the control group. (Fig. 4B). Moderate acid phosphatase activities were found during the late prepupal stage (Fig. 4C).

Yellow bodies isolated from control group showed rather low expression levels of EcR A (Fig. 5a), while, EcR $\mathrm{B} 1$ transcripts reached the third peak level and its relative abundance was 60-fold greater than EcR A (Fig. 5b). While USP 1 levels were low during the late prepupal stage (Fig. $5 \mathrm{c})$ the highest USP 2 levels were detected at this stage (Fig. 5d). The expressions of most of the transcription factors studied, E74 isoforms (Fig. 5e, f), E75 isoforms (Fig. 5g, h), BR-C Z1 (Fig. 6a) and BFTZ-F1 (Fig. 6d), were upregulated on day 10 of the $5^{\text {th }}$ larval instar. On the other hand, BR-C Z2 (Fig. 6b) and BR-C Z4 (Fig. 6c) transcripts decreased and the levels of BHR3 mRNA were almost undetectable (Fig. 6e).

In the yellow bodies isolated from fenoxycarb treated insects, the expression of EcR A was low during the late prepupal stage (Fig. 5a) and the expression of EcR B1 was completely inhibited (Fig. 5b). USP $1 \mathrm{mRNA}$ levels were similar to the control group (Fig. 5c), although, USP 2 transcripts were significantly lower (Fig. 5d). As in the control group, E74A transcripts peaked on day 13 of the $5^{\text {th }}$ larval instar (Fig. 5e) but the peak of E74B in the late prepupal stage was suppressed (Fig. 5f). In addition to this, the peaks of E75 isoforms were also inhibited (Fig. $5 \mathrm{~g}$, h). Interestingly, unlike the control group insects, BR-C Z2 (Fig. 6b), BR-C Z4 (Fig. 6c) and BHR3 (Fig. 6e) mRNA levels were significantly higher. On the other hand, the expression of $\beta$ FTZ-F1 (Fig. 6d) was not significantly different from that recorded for the control insects.

\section{Early pupal stage}

Degeneration of the yellow body proceeded during the early hours of the pupal stage (Fig. 1). In the midguts of the control group, the percentage of TUNEL positive cells peaked at pupation $(29.3 \%)$ then began to decrease gradually after the larval-pupal ecdysis up to $24 \mathrm{~h}$ after pupation (8.1\%) (Fig. 2). The relative abundance of cleaved caspase 3 also declined. (Fig. 3A, C). ATG8 mRNA levels were high during the early hours of the pupal stage (Fig. 4A), however, ATG 12 transcripts were low (Fig. 4B). Acid phosphatase enzyme activity tended to decrease throughout the early pupal stage (Fig. 4C). In the yellow bodies isolated from fenoxycarb treated insects, nearly $38 \%$ of the cells were TUNEL positive up to $24 \mathrm{~h}$ after pupation and then sharply decreased (8.4\%) (Fig. 2). Similarly, cleaved caspase 3 also decreased to almost undetectable levels (Fig. 3B, D). In contrast to the control, low expression of ATG 8 started to increase after the larval-pupal ecdysis 
(Fig. 4A) although ATG 12 mRNA levels remained low during the early pupal stage (Fig. 4B). Like ATG expression, acid phosphatase activity began to increase $12 \mathrm{~h}$ after pupation (Fig. 4C).

During the early pupal stage, EcR A (Fig. 5a) and USP2 (Fig. 5d) mRNA levels were very low in the yellow bodies of control insects and EcR B1 (Fig. 5b) and USP1 (Fig. 5c) transcripts were almost undetectable. At this time only E74 isoforms (Fig. 5e, f) and BFTZ-F1 (Fig. 6d) transcripts were detected. E74B transcripts (Fig. 5f) were almost 20fold higher than those of E74A (Fig. 5e). E75 isoforms (Fig. 5g, h), BR-C isoforms (Fig. 6a, b, c) and BHR3 (Fig. 6e) transcripts were not detected in the early pupal stage.

Similar results were obtained for the yellow bodies from treated insects for EcR isoforms (Fig. 5a, b), USP isoforms (Fig. 5c, d), E75 isoforms (Fig. 5g, h), BR-C Z1 (Fig. 6a), BR-C Z4 (Fig. 6c) and BHR3 (Fig. 6e). E74A mRNA levels were lower than those recorded for the control (fig. 5e), however, E74B (Fig. 5f), BR-C Z2 (Fig. 6b) and BFTZ-F1 (Fig. 6d) mRNA levels were 2.6, 160 and 8.7 fold higher than in the control insects, respectively.

\section{DISCUSSION}

Although the molecular aspects of the role of ecdysone during midgut remodeling in different insect species has been examined in detail, information on the molecular actions of $\mathrm{JH}$ during this process is limited. $\mathrm{JH}$ analogue fenoxycarb treatment on day 0 of the 5th larval instar does not completely prevent PCD but causes a delay by extending the feeding stage (Goncu \& Parlak, 2011) and according to Kamimura \& Kiuchi (1998) this condition is caused by delayed release of ecdysone from the prothoracic gland. In this study, we report the effects of the juvenile hormone analogue fenoxycarb on the expression patterns of ecdysone-regulated genes during programmed cell death of larval midgut of Bombyx mori.

The translocation of cytochrome $\mathrm{c}$ into the cytosol is the first sign of apoptosis occurring in control insects. However, in the fenoxycarb treated insects, cytosolic cytochrome $\mathrm{c}$ was detected most abundantly just before the end of feeding. Wigglesworth (1963) reported that JH causes a decrease in cytochrome $\mathrm{c}$ in different tissues of Rhodnius prolixus. Similarly, mitochondrial swelling occurs in the salivary gland of $D$. melanogaster after JH application. In the light of these findings fenoxycarb application may disrupt the mitochondrial membrane and cause the earlier translocation of cytochrome $\mathrm{c}$ into the cytosol.

Similar to vertebrate nuclear receptors, the heterodimeric ecdysone receptor complex (EcR/USP) acts as a ligand dependent transcription factor and the type of ecdysone receptor isoform in target tissue is important in determining the ultimate response to 20E (Talbot et al., 1993). Fenoxycarb application did not completely inhibit the expressions of ecdysone receptors but did reduce their mRNA levels. Presence of EcR A during the prolonged feeding stage of fenoxycarb treated insects and its downregulation at the beginning of the prepupal stage strongly support this isoform being involved in the maintenance of the structure of the midgut of larvae. In addition, the delay in the secretion of ecdysone following the application of fenoxycarb prevents the down-regulation in the expression of EcR A. However, the EcR B1 peaks that were detected just before the end of feeding in both groups are possibly an important response to $20 \mathrm{E}$ in the reprogramming of the gene cascade involved in cell death. This result also indicates that the inhibitory effect of fenoxycarb on the expression of EcR B1 during the extended feeding stage is indirect and is terminated by the secretion of ecdysone. Among the USP isoforms, upregulation of the expression of USP 2 in fenoxycarb treated insects indicates that this gene may be a target for juvenile hormone. Jones \& Sharp (1997) reported that USP can bind $\mathrm{JH}$, as a homodimer, and suggest that $\mathrm{JH}$ prevents metamorphosis by binding USP as a receptor. In addition, overexpression of USP in the cells of the lepidopteran Spodoptera frugiperda increases JH activation (Riddiford, 2008). King-Jones \& Thummel (2005) propose that unliganded USP is needed to suppress larvalpupal intermediates in Drosophila and $\mathrm{JH}$ is probably involved in this inhibition. Therefore, high expressions of USP isoforms during the feeding stage probably prevent larval-pupal intermediates occurring in the fenoxycarb treated group. When we consider ours and previous results, USP isoforms may be key components in the interaction between $20 \mathrm{E}$ and JH during PCD in the larval midgut. In addition to USP 2 isoform, increase in E74A and E75A after fenoxycarb treatment suggests an additional role for these isoforms in juvenile hormone actions during larval life. Their presence during the extended feeding stage may also indicate that factor(s) other than ecdysone play a role in the expression of these genes. Previous studies report that the application of another juvenile hormone analogue, methoprene, induces the expression of some E74 isoforms in the midguts of $A$. aegypti (Wu et al., 2006) and expression of E75A in Drosophila S2 cells (Dubrovsky et al., 2004), larval haemocytes of Bombyx mori (Matsumoto et al., 2015) and the $5^{\text {th }}$ larval instar epidermis of Manduca sexta (Zhou et al., 1998).

The BRC gene plays a key role in 20E-regulated metamorphic events, therefore, it could be one of the possible targets of JH (Restifo \& Wilson, 1998). In holometabolous insects, initial expressions of BR-C isoforms occur after a small secretion of $20 \mathrm{E}$ in the absence of JH during the last larval instar. However, the expression of the BR-C Z1 isoform in midguts of fenoxycarb treated insects is not induced by ecdysone. Relatively high mRNA levels of BR-C Z1 in the absence of ecdysone (Kamimura \& Kiuchi, 1998) during the feeding period indicate that its expression does not depend on ecdysone and this isoform is probably important in the regulation of larval midgut epithelium and inhibition of death. Some of the previous studies on other species of insect like Blatta germanica (Huang et al., 2013) and Pyrrhocoris apterus (Konopova et al., 2011) show that $\mathrm{JH}$ induces BR-C expression during young nymphal instars. Therefore, it is likely that the presence of BR-C Z1 mRNA's during the feeding period is due to the inductive effect of fenoxycarb. Unlike BR-C Z1, the lower expres- 
sion of BR-C Z2 and BR-C Z4 at the same stage may be due to fenoxycarb inhibiting the secretion of ecdysone.

$\beta$ FTZ-F1 plays an important role in the response of different tissues to ecdysone and may also be involved in $\mathrm{JH}$ signaling during larval-pupal metamorphosis (Bernardo \& Dubrovsky, 2012). Relatively high expressions of $\beta$ FTZ-F1 during the prolonged feeding period indicates that the presence of ecdysone is not required for the expression of this gene and it may be important for maintaining a healthy larval midgut epithelium. In addition, Dubrovsky et al. (2011) report a relationship between the expressions of E75A and FTZ-F1, which act as a competence factor by loading JH signaling components onto the E75A promoter. Therefore, high levels of E75A mRNA during the extended feeding stage of treated insects may be closely associated with the high expression of $\beta$ FTZ-F1 recorded at that time.

Haemolymph $20 \mathrm{E}$ levels begin to increase after the termination of feeding and gut-purge (Kamimura \& Kiuchi, 1998) and early morphological indicators of programmed cell death, like cell shrinkage, cytoplasm and nuclear condensation, occur during the early prepupal stage. When compared to the control, even after ecdysone release, fenoxycarb treatment significantly reduced early prepupal levels of EcR B1 and USP1. Hiruma et al. (1999) reported that the application of juvenile hormone to cultures of Manduca epidermis does not affect the initial increase in ecdysone receptor isoforms but the high amount of $20 \mathrm{E}$ prevents their upregulation. In addition, they propose that the presence of JH with ecdysone prolongs the half-life of EcR B1. EcR B1 protein occurs in the midguts of fenoxycarb treated insects from day 6 up to day 13 (Goncu \& Parlak, 2012). Based on these previous results, the persistence EcR B1 protein after fenoxycarb application may result in a negative feedback regulation and prevent the early prepupal peak of EcR B1 mRNA. The similarity between the expression profiles of USP 1 and EcR B1 indicate that USP 1 might be a partner protein of EcR B1 (Goncu et al., 2016). Similar to EcR B1, the inhibition of USP 1 is probably caused by the same situation. Our results indicate that the expression of BR-C Z2 is strongly suppressed in the absence of ecdysone during the extended feeding stage of fenoxycarb treated insects and this inhibition terminated when the levels of $20 \mathrm{E}$ were high during the second half of the early prepupal stage. Only the E75B isoform was significantly higher in the control group during the early prepupal stage. Jiang et al. (2000) reported that expressions of E75A and E75B in prepupae are sufficient to suppress the transcription of the death inhibitor gene -diap2- in the salivary gland of $D$. melanogaster. Therefore, a similar relationship between E75B and the inhibitor of the apoptosis gene (IAP) during larval midgut degeneration in Bombyx mori needs to be investigated.

DNA fragmentation, caspase 3 activation, acid phosphatase activity and expression of ATG 8 during the late prepupal period indicate the onset of the execution phase of PCD. Despite the correlation between ATG 8 expression and acid phosphatase activity detected in the control group during the late prepupal stage, there is no similar correla- tion in fenoxycarb treated insects. These results indicate that rising acid phosphatase activity at the end of the early prepupal stage is not related to autophagic activity in mature midgut cells of treated insects. Fenoxycarb application significantly reduced mRNA levels of EcR B1, USP2, E74B, E75A and E75B during this phase. Their expression profiles during the extended feeding stage and early prepupal stage strongly indicate a negative feedback regulation of these levels during the late prepupal stage. In contrast, fenoxycarb treatment induced the expression of BR-C Z2, BR-C Z4 and BHR3 at the same stage of development. According to previous studies on holometabolous insects, high levels of $\mathrm{JH}$ are correlated with a low expression of BR-C and a prolonged expression of them in treated insects during the late prepupal stage may due to high levels of $20 \mathrm{E}$ in the presence of JH (Zhou et al., 1998; Suzuki et al, 2008). According to these results, the patterns in the expression of BRC isoforms are similar to that recorded in M. sexta, D. melanogaster and A. aegypti (Wu et al., 2006) in which the application of juvenile hormone analogues causes their re-expression. Absence of BHR3 transcripts in the midguts of the control group indicates that they do not have a role in PCD of the larval epithelium; however, fenoxycarb induces the expression of BHR3 just before pupation. Siaussat et al. (2004) reported that application of $20 \mathrm{E}$ to the imaginal wing cells of Plodia interpunctella in the presence of JH II induces the expression of the HR3 transcription factor. According to Lan et al. (1999) the presence of USP-2 with EcR B1 and USP-1 inhibits the activation of the MHR3 promoter. Therefore, when considering expression profile of USP2, induction of the expression of BHR3 in treated insects could be related to the reduced level of USP2 transcripts detected during the late prepupal stage of fenoxycarb treated insects.

Degeneration of the larval midgut proceeded during the early pupal stage and most of the genes studied, except E74 isoforms and $\beta F T Z-F 1$, decreased to almost undetectable levels during this period. Interestingly, the levels of E74B, BR-C Z2 and BFTZ-F1 mRNA were significantly higher in fenoxycarb treated insects. Based on the morphological, biochemical and molecular analysis of PCD, only the time at which ATG8 was expressed was delayed until pupation and up-regulated during the early pupal stage. Therefore, the high expression of these genes in fenoxycarb treated insect during the early pupal stage may have initiated the autophagy process in the yellow body.

In conclusion, the effects of fenoxycarb on the expression patterns of genes studied differ in a developmentalstage specific manner (Fig. 6). There could be several reasons for this. Throughout the prolonged feeding stage, the inhibitory effect of fenoxycarb on midgut cell death is due to the delayed release of ecdysone. As a result, the expression of EcR A is prolonged and USP2, E74A E75A, BR-C Z1 and $\beta F T Z-F 1$ genes are overproduced. High expressions of these genes indicate that they are probably juvenile hormone inducible genes or their expression is not affected by the absence of ecdysone. On the other hand, fenoxycarb almost completely inhibits the expression of 
BR-C Z2 and BR-C Z4 during the feeding stage, which may due to fenoxycarb delaying the secretion of ecdysone. That spinning activity and gut purge in fenoxycarb treated insects occurs on day 11 of the $5^{\text {th }}$ larval instar are strong evidences that ecdysone is secreted at that time. In addition, Kamimura \& Kiuchi (1998) showed that the secretion of ecdysone was delayed in their experimental animals after 1 ng fenoxycarb was applied on day 0 of the $5^{\text {th }}$ larval instar. Therefore, the sensitizing of the gene to the ecdysone after fenoxycarb treatment may be responsible for the upregulation of some ecdysone regulated genes such as E75B in the early prepupal stage, and BR-C Z2 and BR-C Z4 in the late prepupal stage. In addition, interaction between different ecdysone regulated genes may also be important for their expression profile. For example, the high expression of BHR3 in fenoxycarb treated insects during the late prepupal stage may due to the simultaneous down-regulation in the expression of USP2. On the other hand levels of the mRNA of some of the genes studied decreased after treatment. Significant decrease in the expression patterns during late prepupal stage of fenoxycarb treated insects are observed for five genes, EcR B1, USP 2, E74B, E75A and $\mathrm{E} 75 \mathrm{~B}$ which may due to a negative feedback regulation of the expressions of these genes or to the presence of juvenile hormone related inhibitory factor(s). Even though the timing of the morphological and biochemical events of the degeneration process, such as cellular degeneration and DNA fragmentation, are similar to those recorded for the controls; ATG8 was only expressed in the early pupal stage in the treated insects. Presence of high E74B, BR-C Z2 and $\beta F T Z-F 1$ mRNA levels at the same time indicate that these genes may associated with the expression of ATG8.

ACKNOWLEDGEMENT. This research was supported by The Scientific \& Technological Research Council of Turkey (111T880) and Research and Application Center of Science and Technology (2012 BİL 010) of the Ege University.

\section{REFERENCES}

BAEHRECKE E.H. 2000: Steroid regulation of programmed cell death during Drosophila development. - Cell Death Different. 7: 1057-1062.

Bergmeyer H.U., Gawehn K. \& Grassl M. 1974: Enzymes as biochemical reagents, acid phosphatase. In Bergmeyer H.U. (ed.): Methods of Enzymatic Analysis. Vol. 1. 2nd ed. Academic Press, New York, pp. 495-496.

Bernardo T.J. \& Dubrovsky E.B. 2012: The Drosophila juvenile hormone receptor candidates methoprene-tolerant (MET) and germ cell-expressed (GCE) utilize a conserved LIXXL motif to bind the FTZ-F1 nuclear receptor. - J. Biol. Chem. 2: 7821-7833.

Denton D., Shravage B., Simin R., Mills K., Berry D.L., BaeHRECKE E.H. \& Kumar S. 2009: Autophagy, not apoptosis, is essential for midgut cell death in Drosophila. - Curr. Biol. 19: $1741-1746$.

Denton D., Shravage B., Simin R., Baehrecke E.H. \& Kumar S. 2010: Larval midgut destruction in Drosophila: Not dependent on caspases but suppressed by the loss of autophagy. - $A u$ tophagy 6: 163-165.

Dubrovsky E.B., Dubrovskaya V.A. \& Berger E.M. 2004: Hormonal regulation and functional role of Drosophila E75A or- phan nuclear receptor in the juvenile hormone signaling pathway. - Dev. Biol. 268: 258-270.

Dubrovsky E.B., Dubrovskaya V.A., Bernardo T., Otte V., DiFILIPPO R. \& BRYAN H. 2011: The Drosophila FTZ-F1 nuclear receptor mediates juvenile hormone activation of E75A gene expression through an intracellular pathway. - J. Biol. Chem. 286: $33689-33700$.

Franzetti E., Huang Z.J., Shi Y.X, Xie K., Deng X.J., Li J.P., Li Q.R., Yang W.Y., Zeng W.N., Casartelli M., Deng H.M., Cappellozza S., Grimaldi A., XIa Q., Feng Q., Cao Y. \& TetTAMANTI G. 2012: Autophagy precedes apoptosis during the remodeling of silkworm larval midgut. - Apoptosis 17: 305324.

Franzetti E., Romanelli D., Caccia S., Cappellozza S., Congiu T., Rajagopalan M., Grimaldi A., De Eguileor M., Casartelli M. \& Tettamanti G. 2015: The midgut of the silkmoth Bombyx mori is able to recycle molecules derived from degeneration of the larval midgut epithelium. — Cell Tissue Res. 361: 509-528.

Gibson U.E., Heid C.A. \& Williams P.M. 1996: A novel method for real time quantitative RT-PCR. - Genome Res. 6: 9951001.

Goncu E. \& ParlaK O. 2012: The effect of the juvenile hormone analog, fenoxycarb, on ecdysone receptor B1 expression in the midgut of Bombyx mori during larval-pupal metamorphosis. Folia Histochem. Cytobiol. 50: 52-57.

GonCU E. \& PARLAK O. 2011: The influence of juvenile hormone analogue, fenoxycarb on the midgut remodeling in Bombyx mori (L., 1758) (Lepidoptera: Bombycidae) during larval-pupal metamorphosis. - Turk. J. Entomol. 35: 179-194.

Goncu E., Uranli R., Selek G. \& Parlak O. 2016: Developmental expression of ecdysone-related genes associated with metamorphic changes during midgut remodeling of silkworm Bombyx mori (Lepidoptera: Bombycidae). - J. Insect Sci. 16: $86,13 \mathrm{pp}$.

Hakim R.S., Caccia S., Loeb M. \& Smagghe G. 2009: Primary culture of insect midgut cells. - In Vitro Cell. Dev. Biol. Anim. 45: $106-110$.

Hiruma K., Shinoda T., Malone F. \& Riddiford L.M. 1999: Juvenile hormone modulates 20-hydroxyecdysone-inducible ecdysone receptor and ultraspiracle gene expression in the tobacco hornworm, Manduca sexta. - Dev. Genes Evol. 209: $18-30$.

Huang J.H., Lozano J. \& Belles X. 2013: Broad-complex functions in postembryonic development of the cockroach Blattella germanica shed new light on the evolution of insect metamorphosis. - Biochim. Biophys. Acta 1830: 2178-2187.

Jiang C., BAehreck E.H. \& Thummel C.S. 1997: Steroid regulated programmed cell death during Drosophila metamorphosis. - Development 22: 4673-4683.

Jiang C., Lamblin A.F., Steller H. \& Thummel C.S. 2000: A steroid-triggered transcriptional hierarchy controls salivary gland cell death during Drosophila metamorphosis. - Mol. Cell 5: 445-455.

JONES G. \& ShaRp P.A. 1997: Ultraspiracle: An invertebrate nuclear receptor for juvenile hormones. - Proc. Natl. Acad. Sci. USA 94: 13499-13503.

KAMimuRA M. \& KiUCHI M. 1998: Effects of a juvenile hormone analog, fenoxycarb, on 5th stadium of the larvae of the silkworm, Bombyx mori (Lepidoptera: Bombycidae). - Appl. Entomol. Zool. 33: 333-338.

King-Jones K. \& Thummel C.S. 2005: Nuclear receptors - a perspective from Drosophila. - Nature Rev. Genet. 6: 311-323.

KHOA D.B. \& TAKEDA M. 2012: Expression of autophagy 8 (Atg8) and its role in the midgut and other organs of the greater wax 
moth, Galleria mellonella, during metamorphic remodeling and under starvation. - Insect Mol. Biol. 21: 473-487.

Konopova B., Smykal V. \& Jindra M. 2011: Common and distinct roles of juvenile hormone signaling genes in metamorphosis of holometabolous and hemimetabolous insects. - PLoS ONE 6(12): e28728, 7 pp.

Lan Q., Hiruma K., Hu X., Jindra M. \& Riddiford L.M. 1999: Activation of a delayed-early gene encoding MHR3 by the ecdysone receptor heterodimer EcR-B1-USP-1 but not by EcR-B1-USP-2. - Mol. Cell. Biol. 19: 4897-4906.

Lee C.Y., Simon C.R., Woodard C.T. \& Baehrecke E.H. 2002: Genetic mechanism for the stage-and tissue-specific regulation of steroid triggered programmed cell death in Drosophila. Devel. Biol. 252: 138-148.

Lockshin R.A. \& Williams C.M. 1965: Programmed cell death - I. Cytology of degeneration in the intersegmental muscles of the silkmoth. - J. Insect Physiol. 11: 123-133.

Matsumoto H., Ueno C., NaKamura Y., Kinjoh T., Ito Y., Shimura S., Noda H., Imanishi S., Mita K., Fujiwara H., Hiruma K., Shinoda T. \& Kamimura M. 2015: Identification of two juvenile hormone inducible transcription factors from the silkworm, Bombyx mori. - J. Insect Physiol. 80: 31-41.

NishIURA J.T. 2002: Coordinated morphological changes in midgut, imaginal discs, and respiratory trumpets during metamorphosis of Aedes aegypti (Diptera: Culicidae). — Ann. Entomol. Soc. Am. 95: 498-505.

Nishiura J.T., RaY K. \& MurRay J. 2005: Expression of nuclear receptor - transcription factor genes during Aedes aegypti midgut metamorphosis and the effect of methoprene on expression. - Insect Biochem. Mol. Biol. 35: 561-573.

Parthasarathy R. \& Palli S.R. 2007: Stage- and cell-specific expression of ecdysone receptors and ecdysone-induced transcription factors during midgut remodeling in the yellow fever mosquito, Aedes aegypti. - J. Insect Physiol. 53: 216-229.

Parthasarathy R. \& Palli S.R. 2009: Molecular analysis of juvenile hormone analog action in controlling the metamorphosis of the red flour beetle, Tribolium castaneum. - Arch. Insect Biochem. Physiol. 70: 57-70.

RESTIFo L.L. \& WiLson T.G. 1998: A juvenile hormone agonist reveals distinct developmental pathways mediated by ecdysoneinducible Broad complex transcription factors. - Dev. Genet. 22: $141-159$.

RIDDIFORD L.M. 2008: Juvenile hormone action: A 2007 perspective. - J. Insect Physiol. 54: 895-901.
Santhanam A., Peng W.H., Yu Y.T., SAng T.K., Chen G.C. \& Meng T.C. 2014: Ecdysone-induced receptor tyrosine phosphatase PTP52F regulates Drosophila midgut histolysis by enhancement of autophagy and apoptosis. - Mol. Cell. Biol. 34: 1594-1606.

Siaussat D., Bozzolan F., Queguiner I., Porcheron P. \& DeberNARD S. 2004: Effects of juvenile hormone on 20-hydroxyecdysone-inducible EcR, HR3, E75 gene expression in imaginal wing cells of Plodia interpunctella (Lepidoptera). - Eur. J. Biochem. 271: 3017-3027.

Smagghe G., Vanhassel W., Moeremans C., Wilde D., Goto S., Loeb M.J., Blacburn M.B. \& Hakim R.S. 2005: Stimulation of midgut stem cell proliferation and differentiation by insect hormones and peptides. - Ann. N. Y. Acad. Sci. 1040: 472-475.

SuZUKI Y., TRUMAN J.W. \& RidDiford L.M. 2008: The role of Broad in the development of Tribolium castaneum: Implications for the evolution of the holometabolous insect pupa. Development 135: 569-577.

Talbot W.S., Swyryd E.A. \& Hogness D.S. 1993: Drosophila tissues with different metamorphic responses to ecdysone express different ecdysone receptor isoforms. - Cell 73: 1323-1337.

Tereshima J., Yasuhara N., Iwami M. \& Sakurai S. 2000: Programmed cell death triggered by insect steroid hormone, 20-hydroxyecdysone in the anterior silk gland of the silkworm, Bombyx mori. - Dev. Genes Evol. 210: 545-558.

Tettamanti G., Grimaldi A., Casartelli M., Ambrosetti E., Ponti B., Congiu T., Ferrarese F., Rivas-Pena M.R., PennacChio F. \& De Eguileor M. 2007: Programmed cell death and stem cell differentiation are responsible for midgut replacement in Heliothis virescens during prepupal instar. - Cell Tissue Res. 330: 345-359.

WigGLESWORTH V.B. 1963: The action of moulting hormone and juvenile hormone at the cellular level in Rhodnius prolixus. J. Exp. Biol. 40: 231-245.

Wu Y., Parthasarathy R., Bai H. \& Pallit S.R. 2006: Mechanisms of midgut remodeling: Juvenile hormone analog methoprene blocks midgut metamorphosis by modulating ecdysone action. - Mechan. Dev. 123: 530-547.

Zhou B., Hiruma K., Jindra M., Shinoda T., Segraves W.A., MALONE F. \& Riddiford L.M. 1998: Regulation of the transcription factor E75 by 20-hydroxyecdysone and juvenile hormone in the epidermis of the tobacco hornworm, Manduca sexta, during larval molting and metamorphosis. - Dev. Biol. 193: $127-138$.

Received November 28, 2016; revised and accepted March 27, 2017 Published online May 5, 2017 